\title{
Entrapment as a mediator of suicide crises
}

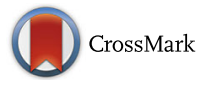

\author{
Shuang Li ${ }^{\dagger}$, Zimri S. Yaseen ${ }^{\dagger}$, Hae-Joon Kim, Jessica Briggs, Molly Duffy, Anna Frechette-Hagan, Lisa J. Cohen \\ and Igor I. Galynker*
}

\begin{abstract}
Background: Prior research has validated the construct of a suicide crisis syndrome (SCS), a specific psychological state that precedes and may precipitate suicidal behavior. The feeling of entrapment is a central concept of the SCS as well as of several other recent models of suicide. However, its exact relationship with suicidality is not fully understood. In efforts to clarify the exact role of entrapment in the suicidal process, we have examined if entrapment mediates the relationship of other components of the SCS, including ruminative flooding, panic-dissociation, fear of dying and emotional pain, with suicidal ideation (SI) in recently hospitalized psychiatric inpatients.

Methods: The Suicide Crisis Inventory (SCI) and Beck Scale for Suicidal Ideation (BSS) were administered to 200 high-risk adult psychiatric inpatients hospitalized following SI or suicide attempt, assessing SCS and SI levels at admission, respectively. The possible mediation effects of entrapment on the relationship between the other components of the SCS and SI at admission were evaluated.

Results: Entrapment significantly and fully mediated the relationship of ruminative flooding, panic-dissociation, and fear of dying with SI, with no direct relationships between these variables and SI reaching statistical significance. Further, no reverse mediation relationships between these variables and SI were found, indicating that the mediation effects of entrapment were unidirectional. While entrapment did mediate the association between emotional pain and $\mathrm{SI}$, the direct relationship between emotional pain and SI was also significant. Moreover, in reverse mediational analysis, emotional pain was a partial mediator of the relationship between entrapment and SI.
\end{abstract}

Conclusion: Entrapment and emotional pain may have a more direct association with $\mathrm{SI}$ than the other components of the SCS, including ruminative flooding, panic-dissociation, and fear of dying, the effects of which are mediated by the former. This suggests entrapment and emotional pain may represent key symptomatic targets for intervention in acutely suicidal individuals. Further research is needed to determine the relationship of these constructs to suicidal behavior.

Keywords: Suicide, Suicidal ideation, Entrapment, Emotional pain, Suicide crisis

\section{Background}

Suicide is a major public health concern, ranking as the 10th leading cause of death in the United States, and accounts for over 42,773 deaths in the US each year [1]. Suicide is a complex behavior reflecting a multi-level interplay of biological, psychological, social, and material factors such as access to means. Although the predictive value of long-term risk factors including biomarkers (e.g., serotonin levels), demographic factors (sex, age), and clinical factors (diagnosis of mental illness, prior

\footnotetext{
* Correspondence: Igor.Galynker@mountsinai.org

${ }^{\dagger}$ Equal contributors

Department of Psychiatry, Beth Israel Medical Center NY, 1st Avenue and 15th Street, New York, NY 10003, USA
}

suicide attempt) has been well established [2, 3], a recent meta-analysis of 50 years of research on risk factors for suicidal thoughts and behaviors (STB) highlights the consistently small effect sizes of such risk factors and that no risk factor category or subcategory is substantially stronger than any other [4]. Likewise, reliable predictors of acute suicide risk have also remained elusive. Recently, however, a small but growing body of evidence suggests the existence of an acute suicide crisis syndrome (SCS) $[5,6]$, characterized by the experience of entrapment accompanied by intense negative affect, loss of cognitive control, hyper-arousal, and social withdrawal, which may precipitate the transition from 
chronic suicidal ideation (SI) to acute suicide attempt (SA) [7-12]. Our previous work on a series of instruments aiming to define the SCS has shown several clinical factors, proposed as criterion symptoms of a clinical syndrome that characterizes the suicidal crisis, are indeed related to each other and short-term risk for suicidal behavior. These include the constructs of entrapment, ruminative flooding (a cognitive control symptom), panic-dissociation and fear of dying (hyper-arousal symptoms), and emotional pain (an intense negative affect symptom) [10-13] and encompass the affective, cognitive, and somatic aspects of the hypothesized SCS. Notably, the Suicide Crisis Inventory (SCI) has demonstrated strong internal consistency, and significant predictive as well as incremental predictive validity over standard suicide predictors (SI, depression, state and trait anxiety) for short-term post-discharge suicidal behavior [12].

The first construct, entrapment, has recently been proposed as a key psychological element of several models of suicidal behavior, notably the Arrested Flight/Cry of Pain model by Williams and Pollock [14] and O'Connor's Integrated Motivational-Volitional Theory (IMV) [15]. According to these models, entrapment, defined as a felt urgency to escape from an unbearable situation from which there is no perceived escape [16], could be a core psychological mechanism in causal pathways to suicide. In agreement with this hypothesis, a strong positive association was found between perceptions of entrapment and suicidality in a range of subacute outpatient populations [17-23]. O'Connor's group also demonstrated that entrapment added incremental predictive validity for suicidal behaviors over depression, hopelessness, SI, and the frequency of previous suicide attempts [24]. Similar to their findings, in our group's latest study of the SCS, entrapment was the strongest individual predictor of suicidal behavior within the 2 months following discharge from an inpatient unit [12].

Surprisingly, although entrapment is a core concept of many models of suicide and of the hypothesized SCS, its role as a possible mediator of the relationship between other acute risk factors and SI/suicidal behavior has never been examined in acutely suicidal patients. The IMV model [15] posits that ruminations increase the likelihood of stressful experiences leading to perceptions of entrapment, which in turn may trigger SI. Alternatively, entrapment may lead to a cognitive search for escape routes resulting in rumination, thus triggering SI. Uncovering these relationships may not only help clarify theoretical models of suicidal behavior, but also establish important targets for therapeutic interventions in acutely suicidal individuals.

The second hypothesized component of the SCS, ruminative flooding, is characterized by uncontrollable perseverative thinking involving continual thoughts about the causes, meaning, and consequences of one's negative mood. Ruminative flooding differs from simple ruminations in that it is experienced as an uncontrollable and overwhelming profusion of negative thoughts, often associated with somatic symptoms inside the head, such as headaches or head pressure [10]. Similar constructs have been associated with elevated suicide risk by other research teams $[25,26]$. The third construct, panicdissociation, describes somatic symptoms commonly associated with a panic-like dissociative state, and involves somatic experiences of unfamiliar sensations felt all over the body, and derealization [11, 13]. Similar constructs have also been described by other suicide researchers $[27$, 28]. The fourth construct, fear of dying, describes morbid cognitions during panic, which may mediate the transition from latent SI to active SI and suicide attempt in some depressed subjects [29-34].

Finally, the fifth hypothesized component of the SCS is emotional pain, a mixture of intense and painfully felt negative emotions such as guilt, shame, hopelessness, disgrace, rage, and defeat, which arises when the essential needs to love, to have control, to protect one's self image, to avoid shame, guilt, and humiliation, or to feel secure are frustrated [35]. Emotional pain is similar to entrapment in that it is strongly correlated with but distinct from anxiety and depression, and can be so intense that the individual seeks to escape by - suicide [35-39]. However, those in emotional pain may lack the desperation caused by the perception that all escape routes are blocked, whereas this desperation and subsequent escape motivation are intrinsic components of entrapment.

Severe or pervasive SI is consistently found to be a significant predictor of subsequent suicidal behaviors [40]. Even passive ideation, such as a wish to die, has been identified as a risk factor for death by suicide [41]. Although the relationship between SI, suicidal behavior, and suicide is not straightforward, and several other symptom dimensions may synergistically influence suicide risk [20, 42], SI has a much higher base rate than suicides or suicide attempts and thus represents a scientifically useful and clinically meaningful modifiable element of suicide risk.

Given the evidence of the central role of entrapment in the suicidal process, the goal of the present report was to establish whether entrapment mediates the relationship between the other clinical factors of the SCS and SI in psychiatric inpatients recently hospitalized for suicide risk. As, the SCS is conceptualized as a clinical syndrome that activates SI, motivating the transition from SI to SA, in the context of the current study, severity of SI serves as a proxy for nearness to suicidal action. We therefore reanalyze the data from our published validation study of the SCI [12] to examine the 
interrelationships among the SCI subscales and severity of suicidal ideation. We hypothesized that entrapment will mediate the relationships between SI and ruminative flooding, panic-dissociation, fear of dying, and emotional pain.

\section{Methods}

\section{Study subjects}

Participants between the ages of 18-65 hospitalized for SI or SA were recruited from the inpatient psychiatric units at Mount Sinai Beth Israel (MSBI) in New York City between April 2013 and July 2015. Potential participants were identified and referred to the study by a clinician. Within $72 \mathrm{~h}$ of observation and admission to the psychiatric inpatient unit, a trained research assistant approached each eligible patient to explain the study, its aims, and the risks and benefits of participation. To avoid reporting bias that might be associated with presenting the study as explicitly focused on suicidality, the study was titled "Predicting Emotional Dysregulation: Internal Consistency and Predictive Validity of the Emotional Dysregulation Scale" and the phrases "SI" and "suicide attempt" were replaced with the term "emotional dysregulation" in the consent form. Following an explanation of what the study entailed, he or she was then presented with a consent form to review and sign, and study measures were completed concurrently.

Participants were excluded if they had cognitive or linguistic limitations precluding their understanding the consent or research questions, significant medical or neurological disease, or possible delirium that might interfere with study participation or informed consent. The study was approved by the MSBI Institutional Review Board, project \#223-14.

\section{Measures}

The suicide crisis inventory (SCI)

The 49-item SCI, comprises 5 subscales: entrapment (13 items), panic-dissociation (9 items), ruminative flooding (7 items), emotional pain (4 items), and fear of dying (3 items). The remaining items contribute to the total SCI score but are not assigned to a subscale. Items are rated by selfreport on a five-point scale ranging from 'not at all $=0$ ' to 'extremely $=4$ '. Each subscale, as previously reported, demonstrates good to excellent internal consistency in the study sample (entrapment: $\alpha=0.946$; panic-dissociation: $\alpha$ $=0.882$; ruminative flooding: $\alpha=0.892$; emotional pain: $\alpha=$ 0.878 and fear of dying: $\alpha=0.796$ ) [12].

\section{Suicidal ideation (SI)}

Severity of SI was assessed with the Part 1 total score of the Beck Scale for Suicidal Ideation (BSS) [43], a 21-item self-report measure of SI. Scores on each item range from 0 to 2, with higher scores indicating greater severity of SI. Part 1 of the BSS was used because Part 2 is completed only if items 4 and 5 in Part 1 are scored $>0$, and thus total scores were not calculated for the entire sample.

\section{Depression severity}

Severity of depression (exclusive of suicidal ideation) at admission was assessed with the Beck Depression Inventory II (BDI) [44, 45], a 21-item self-report measure of depression with item 9 (suicidal ideation) excluded. Scores on each item range from 0 to 3 , with higher scores indicating greater severity of SI.

\section{Statistical analysis}

To investigate the hypothesis that the relationship between each construct and SI was fully mediated by the perception of entrapment, a simple mediation model was applied, with severity of SI as the dependent variable.

\begin{tabular}{|c|c|c|}
\hline Age (years) & Mean $=35.3$ & $\mathrm{SD}=13.4$ \\
\hline Education (years) & Mean $=13.7$ & $\mathrm{SD}=2.9$ \\
\hline Variable & N & Percent \\
\hline \multicolumn{3}{|l|}{ Gender } \\
\hline Male & 91 & 46.3 \\
\hline Transgender & 2 & 1.0 \\
\hline Female & 107 & 53.7 \\
\hline \multicolumn{3}{|l|}{ Race } \\
\hline Asian & 20 & 10.0 \\
\hline Black & 43 & 21.5 \\
\hline White & 76 & 38.0 \\
\hline Other & 61 & 30.5 \\
\hline Ethnicity (Latino) & 68 & 34.0 \\
\hline \multicolumn{3}{|l|}{ Marital status } \\
\hline Never married & 136 & 68.0 \\
\hline Married & 25 & 12.5 \\
\hline Divorced/widowed/separated & 39 & 19.5 \\
\hline \multicolumn{3}{|l|}{ Diagnosis } \\
\hline Psychotic spectrum disorders & 20 & 10 \\
\hline Bipolar spectrum disorders & 26 & 13 \\
\hline Unipolar depressive disorders & 84 & 42 \\
\hline Other disorders $^{\mathrm{a}}$ & 70 & 35 \\
\hline \multicolumn{3}{|l|}{ Substance use } \\
\hline $\mathrm{EtOH}$ use disorder & 75 & 37.5 \\
\hline Illicit substance use disorder & 97 & 48.5 \\
\hline Any substance use disorder & 120 & 60 \\
\hline
\end{tabular}

${ }^{a}$ Mood disorder not otherwise specified, adjustment disorder, substance-induced disorders, personality disorder only, anxiety disorders 
Table 2 Inter-correlations among study variables $(N=200)$

\begin{tabular}{llllll}
\hline & Entrapment & Panic /Dissociation & Ruminative Flooding & Fear of dying & Emotional Pain \\
\hline Entrapment & 1 & & & & \\
Panic/Dissociation & $.510^{* * *}$ & 1 & 1 & & \\
Ruminative Flooding & $.671^{* * *}$ & $.609^{* * *}$ & $.496^{* * *}$ &. & $.454^{* * *}$ \\
Fear of dying & $.519^{* * *}$ & $.572^{* * *}$ & $.619^{* * *}$ & $.145^{*}$ & 1 \\
Emotional Pain & $.708^{* * *}$ & $.500^{* * *}$ & $.316^{* * *}$ & $.417^{* * *}$ \\
SI & $.422^{* * *}$ & $.228^{* *}$ & & & 1 \\
\hline SI suicidal ideation & & &
\end{tabular}

S/ suicidal ideation

${ }^{*} p<0.05$ (2-tailed), ${ }^{* *} p<0.01$ (2-tailed), ${ }^{* * *} p<0.001$ (2-tailed)

Direct, indirect and total effects together with their bias-corrected $95 \%$ confidence intervals were calculated based on 5000 bootstraps. The mediation effect was assessed by (a) Preacher \& Kelley's [46] Kappasquared test, where $\mathrm{k}^{2}=$ the proportion (ranging from 0 to 1 ) of the maximum possible indirect effect observed and (b) the completely standardized effect size (CS) interpreted relative to zero with larger values indicating larger effect size. In those tests, a bootstrapped 95\% confidence interval (CI) excluding zero indicates statistical significance. The Sobel test was chosen as a conservative confirmation of significance of the mediation effect, with a threshold alpha value of 0.05 for significance. To test the unidirectionality of the mediation effects, 'reversed' mediation models were calculated with entrapment as the independent variable and each of the other SCS constructs as the mediator. If no mediation is found in these reversed models, a unidirectional mediation effect of entrapment is supported. In other words, if a mediation effect is significant but the reversed mediation is not, we can say with more confidence that (for example) entrapment accounts for the effect of ruminative flooding on SI and not the other way around.

Finally, in supplementary analyses we examined whether a) SI severity as assessed by the BSS and mediation effects differed between patients admitted in the context of recent suicide attempt and patients admitted with SI only, and b) mediation effects were retained when age and concurrent depression severity were controlled for.

All statistical tests were performed using the Statistical Package for the Social Sciences (SPSS), version 21. Mediation analyses were performed using Hayes [47] PROCESS module model 4 for SPSS.

\section{Results \\ Demographics}

Two hundred and one patients met inclusion criteria and provided informed consent. Data from one participant were excluded because the patient did not complete the BSS. Among these, 142 were hospitalized after presenting with SI, while 58 made attempts leading to their hospitalization. Table 1, summarized below, presents the demographic characteristics of the study participants as previously described [12]. The remaining sample of 200 participants was roughly $50 \%$ female and had a racial composition approximately equivalent to that of the general New York City population. Among the participants, nearly half had a unipolar mood disorder, and approximately one quarter had a psychotic or bipolar mood disorder, while roughly a third were diagnosed with other disorders such as an unspecified adjustment disorder. Over half also were diagnosed with a comorbid substance use disorder.

Table 3 Regression coefficients of entrapment and ruminative flooding predicting suicidal ideation

\begin{tabular}{|c|c|c|c|c|c|c|c|c|}
\hline \multicolumn{9}{|l|}{ Predicted variable } \\
\hline \multicolumn{5}{|l|}{ M(entrapment) } & \multicolumn{4}{|c|}{ Y (suicidal ideation) } \\
\hline Predictor variable & & Coeff. & SE & $P$ & & Coeff. & SE & $P$ \\
\hline X (Ruminative Flooding) & $a$ & 1.192 & 0.094 & $<0.001$ & $c^{\prime}$ & 0.023 & 0.033 & 0.494 \\
\hline M (Entrapment) & & - & - & - & $b$ & 0.082 & 0.019 & $<0.001$ \\
\hline Constant & $i_{1}$ & 13.187 & 1.684 & $<0.001$ & $i_{2}$ & 0.387 & 0.504 & 0.443 \\
\hline \multicolumn{5}{|l|}{$R^{2}=0.450$} & \multicolumn{4}{|c|}{$R^{2}=0.180$} \\
\hline \multicolumn{5}{|c|}{$F(1,198)=162.000, p=<0.001$} & \multicolumn{4}{|c|}{$F(2,197)=21.649, p<0.001$} \\
\hline
\end{tabular}

" $a$ " is the regression coefficient predicting entrapment by ruminative flooding

" $b$ " is the coefficient predicting suicidal ideation by entrapment

" $C$ "' is the coefficient predicting suicidal ideation from ruminative flooding independent of entrapment 
Table 4 Total, direct and indirect effects and effects sizes for indirect effects of ruminative flooding on suicidal ideation mediated by entrapment

\begin{tabular}{|c|c|c|c|c|c|c|c|c|c|c|}
\hline & Raw effect & SE & lower & upper & $p$ & $\mathrm{CS}$ & SE & $k^{2}$ & SE & Z Score \\
\hline Indirect effect & 0.097 & 0.023 & 0.054 & 0.142 & $<0.001$ & 0.256 & 0.061 & 0.202 & 0.047 & 4.140 \\
\hline Direct effect & 0.023 & 0.033 & -0.043 & 0.088 & 0.494 & & & & & \\
\hline Total effect & 0.120 & 0.026 & 0.070 & 0.171 & $<0.001$ & & & & & \\
\hline
\end{tabular}

SE standard error, lower lower bound of $95 \%$ confidence interval, upper upper bound of $95 \%$ confidence interval, $C S$ completely standardized effect size, $\kappa^{2}$ Kappa-squared test, $\mathrm{Z}$ score by Sobel test

\section{Inter-correlations among each component of the suicidal crisis syndrome}

Current SI and each of the subscales of the SCI had significant positive correlations with each other with $r$ values ranging from 0.15 to 0.71 . See Table 2 .

\section{Mediation analyses}

\section{Ruminative flooding}

As indicated in Tables 3 and 4, the total effect of ruminative flooding on SI was significant at $p<0.001$ with a standardized regression coefficient of 0.120 . The direct effect of ruminative flooding on SI was not significant (95\% CI including 0). In contrast, the indirect effect of ruminative flooding on SI was significant (95\% CI did not include 0 and Sobel test: $\mathrm{z}=4.140, p<0.001$ ), suggesting that entrapment fully mediates the relationship between ruminative flooding and SI with small to mediate effect size $\left(C S=0.256, \kappa^{2}=0.202\right)$. As indicated in Table 11, the reversed mediation analysis with entrapment as the independent variable and ruminative flooding as the mediator showed no significant indirect effect (Sobel test: $p=0.494$ ). Both the direct effect and the total effect of entrapment on SI were significant. These results show that entrapment fully mediates the relationship between ruminative flooding and SI, and conversely, that ruminative flooding is not a mediator of the relationship between entrapment and SI.

\section{Panic-dissociation}

As shown in Tables 5 and 6, the total effect of panicdissociation on SI was significant at $p=0.001$ with a standardized regression coefficient of 0.077 . The direct effect of panic-dissociation on SI was not significant $(p=0.821)$. In contrast, the indirect effect of panic-dissociation on SI was significant (95\% CI did not include 0 and Sobel test: $\mathrm{z}=4.573, p<0.001$ ). This suggests that the effect of panic-dissociation on SI was fully mediated by entrapment.

As indicated in Table 11, the reversed mediation analysis with entrapment as the independent variable and panic-dissociation as the mediator showed no indirect effect (Sobel test: $p=0.822$ ) while both the direct effect and the total effect of entrapment on SI were significant. This confirms that entrapment fully mediates the relationship between panic-dissociation and SI, and this relationship is unidirectional.

\section{Fear of dying}

As indicated in Tables 7 and 8, the total effect of fear of dying on SI was significant at $p=0.041$ with a standardized regression coefficient effect of 0.117 . The direct effect of fear of dying on SI was not significant as the corresponding confidence interval includes zero. In contrast, the indirect effect of fear of dying on SI was significant (95\% CI did not include 0 and Sobel test: $\mathrm{z}=$ 5.058, $p<0.001)$.

As indicated in Table 11, the reversed mediation analysis with entrapment as the independent variable and fear of dying as the mediator showed no significant indirect effect (Sobel test: $p=0.185$ ). Both the direct effect and the total effect of entrapment on SI were significant. This indicates that entrapment fully mediates the

Table 5 Regression coefficients of entrapment and panic-dissociation predicting suicidal ideation

\begin{tabular}{|c|c|c|c|c|c|c|c|c|}
\hline \multicolumn{9}{|l|}{ Predicted variable } \\
\hline \multicolumn{5}{|l|}{ M (entrapment) } & \multicolumn{4}{|c|}{ Y (suicidal ideation) } \\
\hline Predictor variable & & Coeff. & SE & $P$ & & Coeff. & SE & $P$ \\
\hline$x$ (Panic dissociation) & $a$ & 0.810 & 0.097 & $<0.001$ & $c^{\prime}$ & 0.006 & 0.026 & 0.821 \\
\hline$M$ (Entrapment) & & - & - & - & $b$ & 0.088 & 0.016 & $<0.001$ \\
\hline Constant & $i_{1}$ & 23.164 & 1.408 & $<0.001$ & $i_{2}$ & 0.469 & 0.489 & 0.339 \\
\hline \multicolumn{5}{|l|}{$R^{2}=0.260$} & \multicolumn{4}{|c|}{$R^{2}=0.174$} \\
\hline \multicolumn{5}{|c|}{$F(1,198)=69.432, P=<0.001$} & \multicolumn{4}{|c|}{$F(2,197)=21.394, P<0.001$} \\
\hline
\end{tabular}

" $a$ " is the regression coefficient predicting entrapment by panic-dissociation

" $b$ " is the coefficient predicting suicidal ideation by entrapment

" $c$ "' is the coefficient predicting suicidal ideation from panic-dissociation independent of entrapment 
Table 6 Total, direct and indirect effects and effects sizes for indirect effects of panic-dissociation on suicidal ideation mediated by entrapment

\begin{tabular}{|c|c|c|c|c|c|c|c|c|c|c|}
\hline & Raw effect & SE & lower & upper & $p$ & CS & SE & $k^{2}$ & SE & Z Score \\
\hline Indirect effect & 0.072 & 0.015 & 0.044 & 0.105 & $<0.001$ & 0.211 & 0.0451 & 0.190 & 0.039 & 4.573 \\
\hline Direct effect & 0.006 & 0.026 & -0.045 & 0.056 & 0.821 & & & & & \\
\hline Total effect & 0.077 & 0.024 & 0.031 & 0.124 & 0.001 & & & & & \\
\hline
\end{tabular}

SE standard error, lower lower bound of $95 \%$ confidence interval, upper upper bound of $95 \%$ confidence interval, CS completely standardized effect size, $\kappa^{2}$ Kappa-squared test, Z score by Sobel test

relationship between fear of dying and SI, and fear of dying is not a mediator of the relationship between entrapment and SI.

\section{Emotional pain}

As indicated in Tables 9 and 10, the total effect of emotional pain on SI was significant at $p<0.001$ with a standardized regression coefficient effect of 0.250 . In contrast to the other SCS constructs, the direct effect of emotional pain on SI was significant with a standardized regression coefficient effect of $0.141, p=0.009$. Nevertheless, the indirect effect was also significant (95\% CI did not include 0 and Sobel test: $\mathrm{z}=2.774, p=0.006$ ), indicating that entrapment is a partial mediator of the relationship between emotional pain and SI.

Partial mediation by entrapment was further confirmed by the reversed mediation analyses with entrapment entered as the independent variable and emotional pain as the mediator. As shown in Table 11, while the total effect of entrapment on SI was significant $(p<0.001)$, both the direct effect $(p=0.005)$ and the indirect effect (Sobel test: $p=0.010)$ were also significant, which suggests that the mediation is not unidirectional. Thus, the mediating relationships of emotional pain and entrapment with SI appear to be reciprocal.

\section{Supplementary analyses}

In supplementary analyses, no differences were found in SI between patients admitted with SA and patients admitted with SI, nor were there substantive differences in the strength of mediation effects.
When age and depression severity were added as covariates to the mediation models the mediation effects of entrapment remained significant for each SCI subscale except emotional pain. (See Additional file 1.) Both entrapment and emotional pain, however, were independent predictors of SI (in separate models controlling for depression), and partially mediated the relation between depression severity and SI severity. (See Additional file 2.)

\section{Discussion}

The primary aim of the present study was to test the hypothesis that entrapment mediates the relationships between other constructs of the SCS (ruminative flooding, panic-dissociation, fear of dying, and emotional pain) and SI. To our knowledge, this is the first study to show that entrapment plays a central role in the relationship between the symptoms of the SCS and SI in acutely suicidal individuals. Further, ruminative flooding, panicdissociation, and fear of dying did not significantly mediate the effect of entrapment on SI, indicating that entrapment fully mediated the relationships between each of these constructs and SI. However, entrapment was only a partial mediator of the relationship between emotional pain and SI, and emotional pain was a significant partial mediator of the effect of entrapment on SI. This suggests both common and independent effects of entrapment and emotional pain on SI.

These results are consistent with our previous work, which has documented the prominence of entrapment in several populations of acutely suicidal patients both in the psychiatric emergency department and among

Table 7 Regression coefficients of entrapment and fear of dying predicting suicidal ideation

\begin{tabular}{|c|c|c|c|c|c|c|c|c|}
\hline \multicolumn{9}{|l|}{ Predicted variable } \\
\hline \multicolumn{5}{|l|}{ M(entrapment) } & \multicolumn{4}{|c|}{ Y (suicidal ideation) } \\
\hline Predictor variable & & Coeff. & SE & $P$ & & Coeff. & SE & $P$ \\
\hline$X$ (Fear of dying) & $a$ & 1.959 & 0.229 & $<0.001$ & $c^{\prime}$ & -0.082 & 0.061 & 0.177 \\
\hline M (Entrapment) & & - & - & - & $b$ & 0.102 & 0.016 & $<0.001$ \\
\hline Constant & $i_{1}$ & 22.413 & 1.452 & $<0.001$ & $i_{2}$ & 0.524 & 0.488 & 0.284 \\
\hline \multicolumn{5}{|l|}{$R^{2}=0.270$} & \multicolumn{4}{|c|}{$R^{2}=0.186$} \\
\hline \multicolumn{5}{|c|}{$F(1,198)=73.161, P=<0.001$} & \multicolumn{4}{|c|}{$F(2,197)=22.476, P<0.001$} \\
\hline
\end{tabular}

" $a$ " is the regression coefficient predicting entrapment by fear of dying

" $b$ " is the coefficient predicting suicidal ideation by entrapment

" $c$ "' is the coefficient predicting suicidal ideation from fear of dying independent of entrapment 
Table 8 Total, direct and indirect effects and effects sizes for indirect effects of fear of dying on suicidal ideation mediated by entrapment

\begin{tabular}{|c|c|c|c|c|c|c|c|c|c|c|}
\hline & Raw effect & SE & lower & upper & $p$ & CS & SE & $k^{2}$ & SE & Z Score \\
\hline Indirect effect & 0.199 & 0.040 & 0.129 & 0.286 & $<0.001$ & 0.247 & 0.049 & 0.222 & 0.042 & 5.058 \\
\hline Direct effect & -0.082 & 0.061 & -0.202 & 0.038 & 0.178 & & & & & \\
\hline Total effect & 0.117 & 0.057 & 0.005 & 0.229 & 0.041 & & & & & \\
\hline
\end{tabular}

SE standard error, lower lower bound of $95 \%$ confidence interval, upper upper bound of $95 \%$ confidence interval, CS completely standardized effect size, $\kappa^{2}$ Kappa-squared test, Z score by Sobel test

psychiatrically hospitalized patients [11, 13]. Our data also support the central role of entrapment in the SCS, as assessed by the Suicide Crisis Inventory [12].

Ruminations have been previously linked to SI and suicidal behavior $[25,48,49]$ although prior research has also pointed to an indirect rather than direct effect. Smith, Alloy [50] demonstrated that hopelessness partially mediated the relationship between ruminations and SI, and fully mediated the association between ruminations and duration of suicidality. Notably, Teismann and Forkmann [51] reported no direct relationship between ruminations and SI in two outpatient populations, as the link between the two was fully mediated by entrapment. Our results also show that the relationship between the ruminative flooding subscale of the SCI and $\mathrm{SI}$ is fully mediated by entrapment. This finding complements that by Teismann and Forkmann [51], and suggests that as ruminations during a suicidal crisis become uncontrollable and overwhelming, the perception of being trapped may precipitate SI.

Similarly, although numerous studies have shown that the experience of somatic symptoms, [11, 13, 27, 28, 52] and fear of dying [29-34], particularly in the context of anxiety and panic, predict SI or suicidal behavior, our current study indicates that in the acute suicidal state there are no direct linkages between either somatic symptoms or fear of dying and SI. Our results indicate instead that the somatic symptoms of panic, dissociation, and fear of dying are indirectly linked with SI via a pathway of entrapment. Thus, as with ruminative flooding, experiencing a fear of dying and dissociative symptoms may increase or simply be correlated with the intensity of stressful experiences that result in a sense of entrapment, which in turn might trigger SI.

Our results show that the relationship between emotional pain and SI was only partially mediated by entrapment, indicating a direct link between emotional pain and SI. Furthermore, the finding that the association between entrapment and SI was partially mediated by emotional pain suggests that these two constructs have both a direct and mutually mediatory relationship to SI. It may be that both of these constructs substantially entail the other. Namely, emotional pain may reliably produce the escape motivation that is necessary for entrapment, and the perception of entrapment may necessarily induce emotional pain. This understanding of the findings is consistent with Baumeister's Escape Theory of Suicide [53] which conceptualizes suicide as an escape from otherwise seemingly inescapable painful self-states. Likewise, Shneidman [35] defines emotional pain or mental pain as a compilation of negative emotions (e.g., anxiety, helplessness, and despair) that reflects frustration with one's inability to fulfill basic psychological needs. In Orbach and Mikulincer's formulation, emotional pain includes loss of self-esteem, as well as feelings of failure, abandonment, emotional flooding, and emptiness and, notably, irreversibility [36-39]. Thus, emotional pain as assessed in studies using the Orbach Mikulincer Mental Pain scale, appears to be substantially convergent with the entrapment subscale of the SCI. The emotional pain subscale of the SCI, on the other hand, comprises only items directly descriptive of an

Table 9 Regression coefficients of entrapment and emotional pain predicting suicidal ideation

\begin{tabular}{|c|c|c|c|c|c|c|c|c|}
\hline \multicolumn{9}{|l|}{ Predicted variable } \\
\hline \multicolumn{5}{|l|}{ M (entrapment) } & \multicolumn{4}{|c|}{ Y (suicidal ideation) } \\
\hline Predictor variable & & Coeff. & SE & $P$ & & Coeff. & SE & $P$ \\
\hline$X$ (Emotional pain) & $a$ & 1.986 & 0.141 & $<0.001$ & $c^{\prime}$ & 0.141 & 0.054 & 0.009 \\
\hline M (Entrapment) & & - & - & - & $b$ & 0.055 & 0.019 & 0.005 \\
\hline Constant & $i_{1}$ & 13.358 & 1.525 & $<0.001$ & $i_{2}$ & 0.279 & 0.486 & 0.566 \\
\hline \multicolumn{5}{|l|}{$R^{2}=0.502$} & \multicolumn{4}{|c|}{$R^{2}=0.206$} \\
\hline \multicolumn{5}{|c|}{$F(1,198)=199.332, P=<0.001$} & \multicolumn{4}{|c|}{$F(2,197)=25.549, P<0.001$} \\
\hline
\end{tabular}

" $a$ " is the regression coefficient predicting entrapment by emotional pain

" $b "$ " is the coefficient predicting suicidal ideation by entrapment

" $c$ "' is the coefficient predicting suicidal ideation from emotional pain independent of entrapment 
Table 10 Total, direct and indirect effects and effects sizes for indirect effects of emotional pain on suicidal ideation mediated by entrapment

\begin{tabular}{|c|c|c|c|c|c|c|c|c|c|c|}
\hline & Raw effect & SE & lower & upper & $p$ & $\mathrm{CS}$ & SE & $k^{2}$ & SE & Z Score \\
\hline Indirect effect & 0.108 & 0.042 & 0.027 & 0.193 & 0.006 & 0.181 & 0.070 & 0.140 & 0.052 & 2.774 \\
\hline Direct effect & 0.141 & 0.054 & 0.035 & 0.248 & 0.009 & & & & & \\
\hline Total effect & 0.250 & 0.039 & 0.173 & 0.326 & $<0.001$ & & & & & \\
\hline
\end{tabular}

SE standard error, lower lower bound of $95 \%$ confidence interval, upper upper bound of $95 \%$ confidence interval, CS completely standardized effect size, $\kappa^{2}$ Kappa-squared test, Z score by Sobel test

emotional experience described in terms of pain. Past research has indicated that emotional pain mediates the relationship between perfectionism and suicidality [54], between hopelessness and SI [55], and between nonsuicidal self-injury and suicide risk [56]. Shneidman [35], and later Orbach [36] further suggested that unbearable psychological pain may mediate the relationships between other relevant psychological factors and suicide. Similarly, in Klonsky \& May's recent Three Step Theory of suicide, the combination of pain and hopelessness drives the development of suicidal ideation; our findings for entrapment, which may be understood as a product of emotional pain and hopelessness, are thus also broadly consistent with the three-step theory [57]. Orbach's studies and the strong correlation we found between entrapment and emotional pain suggest that entrapment is an emotionally painful experience. At the same time, however, the aversive experience of emotional pain may drive an urgency for escape which itself is requisite for the experience of entrapment. Our finding in supplementary analyses that controlling for severity of depression in mediation models of entrapment and emotional pain reduced mediation effects to nonsignificance points to the variance common to all three constructs. Nonetheless, entrapment and emotional pain each show incremental prediction of SI with respect to depression severity, highlighting the pertinence of each of these constructs.

Thus, taken altogether, our data indicate that in the SCS, entrapment acts as a conduit between most of the associated symptoms and SI. This finding is highly consistent with recent theoretical models of suicide including the Cry of Pain Model [14, 58] and the IMV Model of suicide [15], as well as Baumeister's Escape Theory of Suicide [53] and Gilbert's phenomenon of 'arrested flight' [16] and Galynker's Narrative Crisis model [6]. Williams and Pollock [14] posit that suicide results from a feeling of defeat in response to humiliation or rejection, which in turn leads to a perception of entrapment. When the latter is combined with a failure to find alternative ways to solve a problem, i.e. cognitive rigidity [59], a suicidal person may see no exit out of his or her perceived entrapment other than suicide. One interpretation of the mediating role of entrapment is that perceptions of entrapment may constitute a final common pathway to SI. Therefore, therapeutic interventions aimed at reducing the perception of entrapment and creating actionable alternatives may eventually reduce suicide risk. In addition, clinical evaluation of entrapment, as illustrated in our previous reports $[11,13]$ by explicitly asking whether a patient felt "trapped," or that "there was no way out," or that he or she "had no good options," would be essential for a suicide risk assessment. Further, our data suggest that in addition to explicitly asking whether patients feel trapped in their current life situation, relationship, or within themselves, it is important to assess whether they have experienced fear of dying, felt unusual sensations in their bodies or pressure inside their heads, and lost the ability to control and stop their ruminative thoughts, as such experiences were robustly correlated with entrapment and may provide a path of approach to assessment of suicidal thoughts that the patient might not otherwise disclose.

Based on our current results, and our findings from the complementary study of prediction of suicidal behavior [12], it can be hypothesized that while either unbearable emotional pain or entrapment may mediate the relationship between other symptoms of the SCS and SI, it is entrapment that may be the final mediator of the relationship of

Table 11 Regression models for mediation of entrapment effects on SI by SCS components (reversed analysis)

\begin{tabular}{llll}
\hline Mediating variable & $\begin{array}{l}\text { Total effect of Entrapment on } \\
\text { Sl: coefficient effect; (T score) }\end{array}$ & $\begin{array}{l}\text { Direct effect of Entrapment on } \\
\text { SI: coefficient effect; (T score) }\end{array}$ & $\begin{array}{l}\text { Indirect effect of Entrapment on SI by Normal } \\
\text { theory test: coefficient effect; (Z score) }\end{array}$ \\
\hline Ruminative flooding & $0.090 ;(6.553)^{* * *}$ & $0.082 ;(4.393)^{* * *}$ & $0.009 ;(0.683)$ \\
Panic-dissociation & $0.090 ;(6.550)^{* * *}$ & $0.088 ;(5.509)^{* * *}$ & $0.002 ;(0.225)$ \\
Fear of dying & $0.090 ;(6.550)^{* * *}$ & $0.102 ;(6.314)^{* * *}$ & $-0.011 ;(-1.327)$ \\
Emotional pain & $0.090 ;(6.553)^{* * *}$ & $0.055 ;(2.836)^{* *}$ & $0.036 ;(2.573)^{*}$
\end{tabular}

$S /$ severity of suicidal ideation, SCS suicide crisis syndrome

${ }^{*} p<0.05,{ }^{* *} p<0.01,{ }^{* * *} p<0.001$ 
multiple psychological factors and suicidal action. A mediation analysis of prospective suicidal behavior using a larger sample size may provide support for this testable hypothesis. As the SCS is proposed to be a syndrome, the strong inter-correlations among the SCI subscales are certainly supportive of the SCS concept. Nonetheless, further study is needed to determine whether such a syndrome is more predictive of STB than simple severity of entrapment.

The results of this study need to be considered in the context of its limitations. This study represents a novel analysis of our previously published scale-validation data and thus all conclusions are tempered by the need for replication. Further, the study examines the relationship between the SCS constructs and SI rather than suicidal behavior or actual suicide, making the results less relevant to the urgent clinical task of suicide prevention. However, both active and passive SI have been shown to predict death by suicide marking it as a significant modifiable element of suicide risk $[40,41]$; thus, understanding the mediation effects of the relationships of psychiatric symptoms in acutely suicidal individuals with SI can improve our understanding of the suicidal process in general. Second, the cross-sectional design of the study limits any causal interpretation of our findings. Third, the current study utilized only a selfreport measure of SI, and the data may be confounded if some participants were not forthcoming in revealing their suicidality. Finally, the study was conducted at a single site, and study findings need to be replicated at other locations.

\section{Conclusion}

Within its limitations, the current study highlights the central links between SI and entrapment and emotional pain in high-risk patients. The results suggest that psychiatric symptoms of a suicide crisis, such as ruminative flooding, panic-dissociation, and fear of dying may result in SI when they are experienced as painful states lacking routes for escape. The study further suggests that for individuals in a state of suicide crisis, the psychological processes tying entrapment and emotional pain to SI are highly similar, and generally consistent with models conceptualizing suicide as an escape behavior. Further research is warranted to establish the respective mediatory roles of entrapment and emotional pain with regard to future suicidal behavior.

\section{Additional files}

Additional file 1: Mediation models controlling for age and depression. Results of mediation analyses controlling for age and depression. (DOC $63 \mathrm{~kb}$ )

Additional file 2: Mediation models demonstrating mutual partial mediation of entrapment and emotional pain by depression. Results of mediation analyses demonstrating mutual partial mediation of entrapment and emotional pain by depression. (DOC $57 \mathrm{~kb}$ )

\section{Abbreviations}

BSS: Beck Scale for Suicide Ideation; IMV: Integrated Motivational-Volitional Model; MSBI: Mount Sinai Beth Israel; SCl: Suicide Crisis Inventory;

SCS: Suicide Crisis Syndrome; SI: Suicidal Ideation; STB: Suicidal Thoughts and Behaviors

\section{Funding}

This work was supported by the focus grant \# RFA-1-015-14 from the American Foundation for Suicide Prevention. The content is solely the responsibility of the authors and does not necessarily represent the official views of the American

Foundation for Suicide Prevention. The funders had no role in study design, data collection and analysis, decision to publish, or preparation of the manuscript.

\section{Availability of data and materials}

The data cannot be shared due to confidentiality issues.

\section{Authors' contributions}

$S L, Z Y$, and IG contributed to the concept and design of the study. JB, MD, and $A F H$ collected the data. SL and ZY analyzed the data. SL, ZY, IG, HK, JB, $M D, A F H$, and LC wrote and revised the manuscript. All authors read and approved the final manuscript.

\section{Ethics approval and consent to participate}

The study was approved by the Mount Sinai Beth Israel Institutional Review Board, project \#223-14. Informed consent was obtained from all participants.

Consent for publication

Not applicable.

Competing interests

The authors declare that they have no competing interests.

\section{Publisher's Note}

Springer Nature remains neutral with regard to jurisdictional claims in published maps and institutional affiliations.

Received: 9 May 2017 Accepted: 1 January 2018

Published online: 08 January 2018

\section{References}

1. Control, C.f.D. and Prevention, WISQARS ${ }^{\mathrm{TM}}$ (web-based injury statistics query and reporting system). 2014

2. Pandey GN, Dwivedi Y. Peripheral biomarkers for suicide. In: Dwivedi Y, editor. The neurobiological basis of suicide. London: CRC Press; 2012. p. 407-24.

3. Suokas J, et al. Long-term risk factors for suicide mortality after attempted suicide-findings of a 14-year follow-up study. Acta Psychiatr Scand. 2001; 104(2):117-21.

4. Franklin JC, et al. Risk factors for suicidal thoughts and behaviors: a metaanalysis of 50 years of research. Psychol Bull. 2017;143(2):187-232.

5. Rogers ML, et al. An overview and comparison of two proposed suicidespecific diagnoses: acute suicidal affective disturbance and suicide crisis syndrome. Psychiatr Ann. 2017:47(8):416-20.

6. Galynker I. The suicidal crisis: clinical guide to the assessment of imminent suicide risk. New York: Oxford University Press; 2017.

7. Fawcett J, et al. Time-related predictors of suicide in major affective disorder. Am J Psychiatry. 1990;147(9):1189.

8. Hendin $\mathrm{H}$, et al. Role of intense affects in predicting short-term risk for suicidal behavior: a prospective study. J Nerv Ment Dis. 2010;198(3):220-5.

9. Hendin H, Maltsberger JT, Szanto K. The role of intense affective states in signaling a suicide crisis. J Nerv Ment Dis. 2007;195(5):363-8.

10. Yaseen $Z$, et al. Construct development: the suicide trigger scale (STS-2), a measure of a hypothesized suicide trigger state. BMC Psychiatry. 2010;10(1):110.

11. Yaseen ZS, et al. Predictive validity of the suicide trigger scale (STS-3) for post-discharge suicide attempt in high-risk psychiatric inpatients. PLoS One. 2014;9(1):e86768.

12. Galynker l, et al. Prediction of suicidal behavior in high risk psychiatric patients using an assessment of acute suicidal state: the suicide crisis inventory. Depress Anxiety. 2017;34(2):147-58. 
13. Yaseen ZS, et al. Emergency room validation of the revised suicide trigger scale (STS-3): a measure of a hypothesized suicide trigger state. PLoS One. 2012;7(9):e45157.

14. Williams JMG, Pollock LR. Psychological aspects of the suicidal process Underst Suicidal Behav. 2001:76-93.

15. O'Connor RC, Cleare S, Eschle S, Wetherall K, Kirtley OJ. The integrated motivational-volitional model of suicidal behavior. In: O'Connor RC, Pirkis J, editors. The international handbook of suicide prevention. Chichester: John Wiley \& Sons, Ltd; 2016. p. 220-40.

16. Gilbert P, Allan S. The role of defeat and entrapment (arrested flight) in depression: an exploration of an evolutionary view. Psychol Med. 1998; 28(3):585-98.

17. Maria P, et al. Negative self-appraisals and suicidal behavior among trauma victims experiencing Ptsd symptoms: the mediating role of defeat and entrapment. Depress Anxiety. 2012;29(3):187-94.

18. Panagioti $\mathrm{M}$, et al. A model of suicidal behavior in posttraumatic stress disorder (PTSD): the mediating role of defeat and entrapment. Psychiatry Res. 2013;209(1):55-9.

19. Panagioti M, Gooding PA, Tarrier N. Hopelessness, defeat, and entrapment in posttraumatic stress disorder: their association with suicidal behavior and severity of depression. J Nerv Ment Dis. 2012;200(8):676-83.

20. Rasmussen SA, et al. Elaborating the cry of pain model of suicidality: testing a psychological model in a sample of first-time and repeat self-harm patients. Br J Clin Psychol. 2010;49(1):15-30.

21. Siddaway $A P$, et al. A meta-analysis of perceptions of defeat and entrapment in depression, anxiety problems, posttraumatic stress disorder, and suicidality. J Affect Disord. 2015;184:149-59.

22. Taylor PJ, et al. Defeat and entrapment in schizophrenia: the relationship with suicidal ideation and positive psychotic symptoms. Psychiatry Res. 2010;178(2):244-8.

23. Taylor PJ, et al. Appraisals and suicidality: the mediating role of defeat and entrapment. Arch Suicide Res. 2010;14(3):236-47.

24. O'connor RC, et al. Psychological processes and repeat suicidal behavior: a four-year prospective study. J Consult Clin Psychol. 2013;81(6):1137.

25. Miranda R, Nolen-Hoeksema S. Brooding and reflection: rumination predicts suicidal ideation at 1-year follow-up in a community sample. Behav Res Ther. 2007;45(12):3088-95.

26. Tucker RP, et al. Rumination and suicidal ideation: the moderating roles of hope and optimism. Personal Individ Differ. 2013;55(5):606-11.

27. Garcia-Campayo J, et al. The reason for medical consultations in patients with psychiatric diseases: somatization phenomena and suicide attempts. Medicina Clinica. 1997;108(9):321-4.

28. Öztürk E, Sar V. Somatization as a predictor of suicidal ideation in dissociative disorders. Psychiatry Clin Neurosci. 2008;62(6):662-8.

29. Bolton $\mathrm{JM}$, et al. Anxiety disorders and risk for suicide attempts: findings from the Baltimore epidemiologic catchment area follow-up study. Depress Anxiety. 2008;25(6):477-81.

30. Capron DW, Lamis DA, Schmidt NB. Test of the depression distress amplification model in young adults with elevated risk of current suicidality. Psychiatry Res. 2014;219(3):531-5.

31. Capron DW, et al. Distress tolerance and anxiety sensitivity cognitive concerns: testing the incremental contributions of affect dysregulation constructs on suicidal ideation and suicide attempt. Behav Ther. 2013;44(3):349-58.

32. Tucker RP, et al. Maladaptive five factor model personality traits associated with borderline personality disorder indirectly affect susceptibility to suicide ideation through increased anxiety sensitivity cognitive concerns. Psychiatry Res. 2016;246:432-7.

33. Yaseen ZS, et al. Fear of dying in panic attacks predicts suicide attempt in comorbid depressive illness: prospective evidence from the national epidemiological survey on alcohol and related conditions. Depress Anxiety. 2013;30(10):930-9.

34. Yaseen ZS, et al. Panic as an independent risk factor for suicide attempt in depressive illness: findings from the National Epidemiological Survey on alcohol and related conditions (NESARC). J Clin Psychiatry. 2011;72(12):1628-35.

35. Shneidman ES. Suicide as psychache: a clinical approach to self-destructive behavior. Northfield: Jason Aronson; 1993.

36. Orbach I. Mental pain and suicide. Isr J Psychiatry Relat Sci. 2003;40(3):191.

37. Orbach I, et al. Mental pain and its relationship to suicidality and life meaning. Suicide Life Threat Behav. 2003;33(3):231-41.

38. Orbach I, et al. Mental pain: a multidimensional operationalization and definition. Suicide Life Threat Behav. 2003:33(3):219-30.
39. Troister T, Holden RR. Factorial differentiation among depression, hopelessness, and psychache in statistically predicting suicidality. Meas Eval Couns Dev. 2013;46(1):50-63.

40. Brown GK, et al. Risk factors for suicide in psychiatric outpatients: a 20-year prospective study. J Consult Clin Psychol. 2000;68(3):371.

41. Brown GK, et al. The internal struggle between the wish to die and the wish to live: a risk factor for suicide. Am J Psychiatr. 2005;162(10):1977-9.

42. Yaseen ZS, et al. Functional domains as correlates of suicidality among psychiatric inpatients. J Affect Disord. 2016;203:77-83.

43. Beck AT, Steer RA. BSI, Beck scale for suicide ideation: manual. San Antonio: Psychological Corporation; 1991.

44. Beck AT, Steer RA, Brown GK. Beck depression inventory-II. San Antonio. 1996:78(2):490-8.

45. Steer RA, et al. Mean Beck depression inventory-\|l scores by severity of major depressive episode. Psychol Rep. 2001:88(3_suppl):1075-6.

46. Preacher KJ, Kelley K. Effect size measures for mediation models: quantitative strategies for communicating indirect effects. Psychol Methods. 2011;16(2):93.

47. Hayes AF. Introduction to mediation, moderation, and conditional process analysis: a regression-based approach. New York: Guilford Press; 2013

48. Levi-Belz Y, et al. Mental pain, communication difficulties, and medically serious suicide attempts: a case-control study. Arch Suicide Res. 2014;18(1):74-87.

49. Miranda $\mathrm{R}$, et al. Cognitive inflexibility and suicidal ideation: mediating role of brooding and hopelessness. Psychiatry Res. 2013;210(1):174-81.

50. Smith JM, Alloy LB, Abramson LY. Cognitive vulnerability to depression, rumination, hopelessness, and suicidal ideation: multiple pathways to selfinjurious thinking. Suicide Life Threat Behav. 2006;36(4):443-54.

51. Teismann T, Forkmann T. Rumination, entrapment and suicide ideation: a mediational model. Clin Psychol Psychother. 2017;24(1):226-34.

52. Rappaport LM, et al. Panic symptom clusters differentially predict suicide ideation and attempt. Compr Psychiatry. 2014;55(4):762-9.

53. Baumeister RF. Suicide as escape from self. Psychol Rev. 1990;97(1):90.

54. Flamenbaum R, Holden RR. Psychache as a mediator in the relationship between perfectionism and suicidality. J Couns Psychol. 2007;54(1):51.

55. Flamenbaum, R., Testing Shneidman's theory of suicide: Psychache as a prospective predictor of suicidality and comparison with hopelessness. 2009.

56. Nahaliel $\mathrm{S}$, et al. Mental pain as a mediator of suicidal tendency: a path analysis. Compr Psychiatry. 2014;55(4):944-51.

57. Klonsky ED, May AM. The three-step theory (3ST): a new theory of suicide rooted in the "ideation-to-action" framework. Int J Cogn Ther. 2015;8(2):114-29.

58. Williams M. Cry of pain: understanding suicide and the suicidal mind. London: Little, Brown Book Group; 2014.

59. Schotte DE, Clum GA. Problem-solving skills in suicidal psychiatric patients. Consult Clin Psychol. 1987:55(1):49.

\section{Submit your next manuscript to BioMed Central and we will help you at every step:}

- We accept pre-submission inquiries

- Our selector tool helps you to find the most relevant journal

- We provide round the clock customer support

- Convenient online submission

- Thorough peer review

- Inclusion in PubMed and all major indexing services

- Maximum visibility for your research

Submit your manuscript at www.biomedcentral.com/submit 